\title{
In Search of Regulators of LeSPL-CNR by South-Western Blotting and Yeast One-Hybrid Library Screening System
}

\author{
Xiaohong Wang1, Bishun Ye1, Ying Wang1, Ting Zhou ${ }^{2 *}$, Tongfei Lai ${ }^{*}$ \\ ${ }^{1}$ Research Centre for Plant RNA Signaling, College of Life and Environmental Sciences, Hangzhou Normal University, Hangzhou, \\ China \\ ${ }^{2}$ Key Laboratory for Quality and Safety of Agricultural Products of Hangzhou City, College of Life and Environmental Science, \\ Hangzhou Normal University, Hangzhou, China \\ Email: zt20100061@163.com, *laitongfei@163.com
}

How to cite this paper: Wang, X.H., Ye, B.S., Wang, Y., Zhou, T. and Lai, T.F. (2018) In Search of Regulators of LeSPL-CNR by South-Western Blotting and Yeast OneHybrid Library Screening System. American Journal of Plant Sciences, 9, 1037-1050. https://doi.org/10.4236/ajps.2018.95080

Received: March 15, 2018

Accepted: April 17, 2018

Published: April 20, 2018

Copyright $\odot 2018$ by authors and Scientific Research Publishing Inc. This work is licensed under the Creative Commons Attribution International License (CC BY 4.0).

http://creativecommons.org/licenses/by/4.0/

\begin{abstract}
LeSPL-CNR is a crucial transcription factor for fruit ripening of Solanum lycopersicum. The cnr (colorless non-ripening) epimutation resulted from hypermethylation in a $286 \mathrm{bp}$ region of LeSPL-CNR promoter inhibits normal fruit ripening. In present study, potential regulators of LeSPL-CNR, which could bind to the specific 286 bp region, were screened via south-western blotting and yeast one-hybrid $(\mathrm{Y} 1 \mathrm{H})$ library screening system. Results indicated that a total of 13 and 19 candidate proteins were acquired respectively, and both ribulose-1,5-bisphosphate carboxylase/oxygenase and 40S ribosomal protein were identified by two methods. These would provide some information for revealing roles of DNA methylation and the regulatory mechanism for $L E S P L-C N R$.
\end{abstract}

\section{Keywords}

Solanum lycopersicum, LeSPL-CNR, 286 bp Region, South-Western Blotting, Yeast One-Hybrid

\section{Introduction}

Fruit ripening is a genetically regulated process involving a series of coordinated biochemical, physical and organoleptic changes in color, flavor, texture, aroma, and nutritional status, which is controlled by endogenous hormonal, genetic regulators and environmental stimuli [1] [2] [3]. To understand biological mechanisms of fruit ripening, tomato (Solanum lycopersicum) has been studied 
most extensively, in view of biological advantages of this model system such as well-known structural genomics, rich transcriptome and proteome databases, amenability to genetic transformation, and short life cycle [4] [5]. Solanum lycopersicum cv. Ailsa Craig (AC) with a medium size has been cultivated for around 60 years. It can be grown indoors or out and produces a regular looking fruit which is grown on heavy trusses. In addition, AC has good disease resistance which makes it a nice reliable variety. Therefore, it usually serves as a background for derived-mutants or transgenic lines.

Considerable progress has been made in elucidating the biochemical and molecular basis of fruit ripening that has established the important role of ethylene during fruit ripening in tomato [6] [7] [8] [9] [10]. Fruit-specific transcriptional control of ripening as well as crosstalk among the already characterized components in tomato also has received considerable attention [11] [12]. For example, LeMADS-RIN, LeSPL-CNR, SIFUL1/SIFUL2, LeHB-1, SITAGL1, SIMADS1, SIAP2a, SINACA and SIZFP2 all play crucial roles in tomato fruit ripening by both ethylene-dependent and independent pathways [13]-[21].

Recent studies have suggested that epigenetic changes play pivotal roles in plant development and fruit ripening progress [22] [23]. Liu et al. [24] showed a direct cause and effect relationship between active DNA demethylation (mainly mediated by tomato DEMETER-like DNA demethylases) and fruit ripening. Zhong et al. [25] found that the epigenome of tomato was dynamic, and the global methylation level gradually declined during wild type fruit development and ripening but remained high in cnr (colourless non-ripening) and rin ( $r i$ pening inhibitor) mutants. As a dominant pleiotropic mutation, the cnr epimutation results from a naturally occurring hypermethylation in a $286 \mathrm{bp}$ region of LeSPL-CNR promoter. The cnr mutant greatly inhibits ethylene production and softening, and possesses a non-ripening phenotype with a yellow skin and a colorless mealy pericarp (Figure 1(a) and Figure 1(b)) [13] [26]. The distinct phenotype is not reversed by exposure to exogenous ethylene. In cnr mutant, the biosynthesis of ripening-related pericarp carotenoid is abolished due to the reduced ability to synthesize the carotenoid precursor geranylgeranyl diphosphate (GGPP) [27]. Meanwhile, the solubilisation of homogalacturonan-rich pectic polysaccharides and the proportion of carbohydrate are less than those in wild type. Those lead to reduced cell-to-cell adhesion which reflects changes in the structure of $c n r$ cell walls [28]. LeSPL-CNR is mapped in the euchromatin region on the long arm of tomato chromosome 2 and encodes a transcription factor of the Squamosa Promoter Binding-like Protein. Evidences indicated that a sophisticated regulatory mechanism existed at the upstream of $L e S P L-C N R$. Besides the binding site of LeMADS-RIN in the promoter of LeSPL-CNR [18], the 3'-UTR also possessed a potential $m i R N A 156 / 7$ target. SlymiR157 could regulate the expression of $L e S P L-C N R$ in a likely dose-dependent manner through miRNA-induced mRNA degradation and translation repression [29]. Meanwhile, infection of tomato plants with cucumber mosaic virus (CMV) carrying 

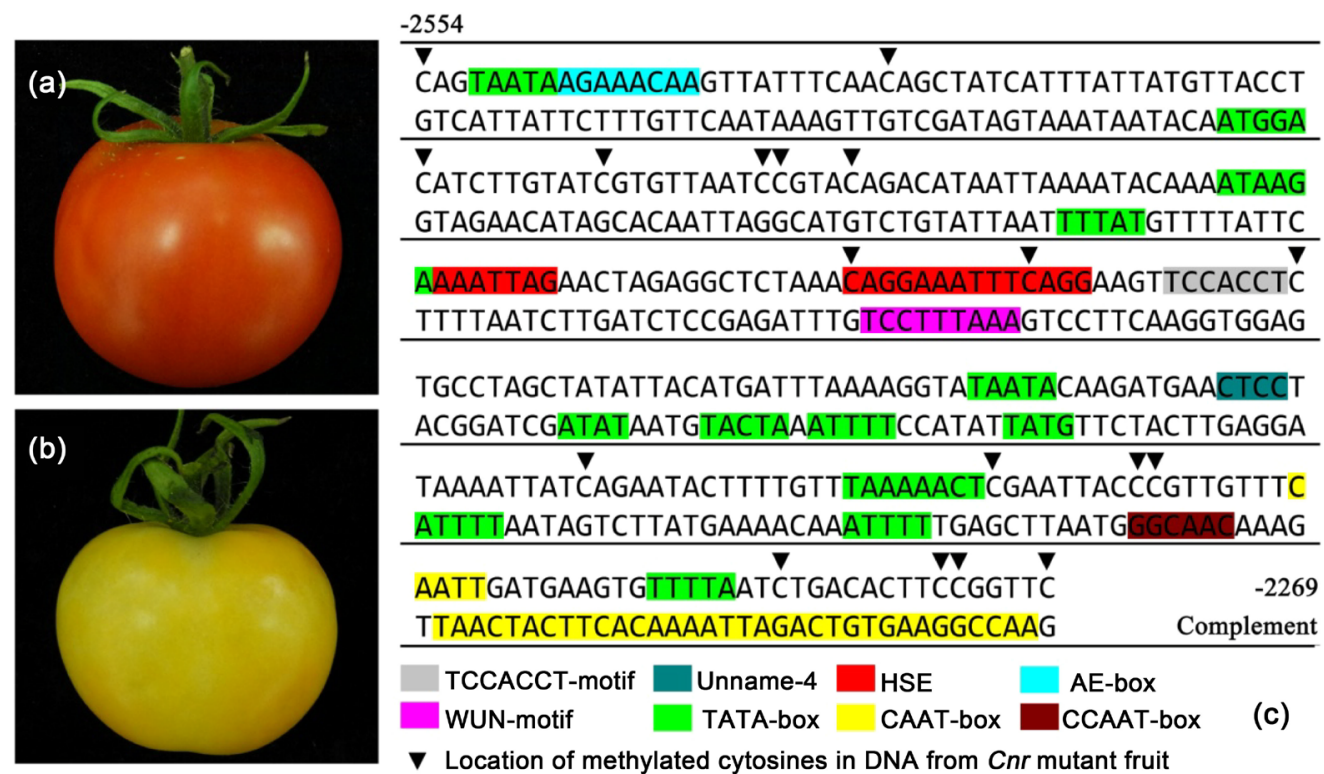

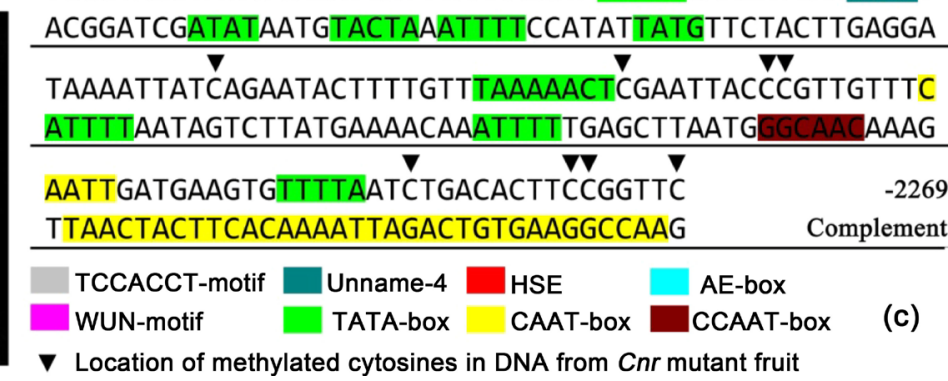

Figure 1. Ripening phenotypes of wild-type AC (a) and cnr mutant fruits (b) with structural and methylation site analysis of a 286 bp region in $L E S P L-C N R$ promoter (c).

the LeSPL-CNR promoter could induce epigenetic changes and result in inhibition of fruit ripening and mottled phenotype [30].

In this context, we surmised that the 286 bp region of LeSPL-CNR promoter contained some valuable sites. The hypermethylation of this region could prevent the binding of some potential regulators, and these regulators might play a critical role in complicated transcriptional cascade or epigenetic controls for fruit ripening. Thus, a screening via south-western blotting and yeast one-hybrid library screening system was carried on to find these potential regulators in present study. Results would provide some information to reveal roles of DNA methylation in plant genomes and elucidate the regulating mechanism for LeSPL-CNR.

\section{Materials and Methods}

\subsection{Plant Materials and Cis-Acting Regulatory Element Analysis}

Solanum lycopersicum cv. Ailsa Craig (AC) and the cnr mutant were grown in glasshouses at $25^{\circ} \mathrm{C}$ and $80 \%$ humidity with a photoperiod of $16 \mathrm{~h}$ day $/ 8 \mathrm{~h}$ night. Tomato flowers were tagged at anthesis and fruit development and ripening stages were recorded as days post anthesis (DPA). In silico analysis of the $286 \mathrm{bp}$ region of LeSPL-CNR promoter were performed in PlantCARE which was a database of plant cis-acting regulatory elements, enhancers and repressors. A motif search algorithm called Motif Sampler and a probabilistic approach based on Gibbs Sampling were used [31]. The PlantCARE relational database was available at http://bioinformatics.psb.ugent.be/webtools/plantcare/html/. Regulatory elements are represented by positional matrices, consensus sequences and individual sites on particular promoter sequences. 


\subsection{Two-Dimensional Electrophoresis and South-Western Blotting}

Total genomic DNA of AC fruits at breaker stage was extracted using a DNeasy Plant Mini Kit (Qiagen, Germany), following the manufacturer's instruction. The 286 bp region was amplified from genome DNA with specific primers (P1F and $\mathrm{P} 1 \mathrm{R}$ in Table 1) and used as a probe in sequent experiment. PCR reactions were conducted in a total volume of $20 \mu \mathrm{L}$ containing $1 \mu \mathrm{L}$ DNA template, 0.5 $\mu \mathrm{L}$ forward primer, $0.5 \mu \mathrm{L}$ reverse primer, $1 \mu \mathrm{L}$ dNTP, $0.1 \mu \mathrm{L}$ PrimeSTAR HS DNA polymerase, $4 \mu \mathrm{L} 5 \times$ PrimeSTAR Buffer $\left(\mathrm{Mg}^{2+}\right.$ plus) and $12.9 \mu \mathrm{L}$ deionized water. PCR conditions for amplification were $94^{\circ} \mathrm{C}$ for $3 \mathrm{~min}$ followed by 25 cycles of $94^{\circ} \mathrm{C}$ for $20 \mathrm{~s}, 53^{\circ} \mathrm{C}$ for $30 \mathrm{~s}$ and $72^{\circ} \mathrm{C}$ for $40 \mathrm{~s}$. Then, a PCR DIG Probe Synthesis Kit (Roche, USA) was used to acquire high labeled and sensitive [digoxigenin(DIG-11)]-dUTP-labelling probe. After purification by High Pure PCR Product Purification Kit (Roche, USA), a series of dilutions of DIG-labeled probe and control DNA were applied to determine the working concentration of labeled probe according to the product manual.

The extraction and quantitation of total cellular proteins of AC fruits at breaker stage used methods described by [32]. About $100 \mu \mathrm{g}$ of proteins were loaded on a rehydrate immobilized $\mathrm{pH}$ gradient gel strip $(7 \mathrm{~cm}, \mathrm{pH} 3$ - 10) (Bio-Rad, USA) and two-dimensional electrophoresis (2-DE) was carried out according to previous reports [33]. Coomassie Brilliant Blue R-250 was used to stain the proteins in a duplicate gel to monitor the equaling of samples. The proteins in one of polyacrylamide gels were transferred to PVDF membrane ( $0.45 \mu \mathrm{m}$, Millipore, USA) by a Trans-Blot Turbo (Bio-Rad, USA). After washing, renaturation, and incubation with DIG-labeled probe, immunological detection using anti-digoxigenin-AP conjugate (Roche, USA) and NBT/BCIP solution (Promega, USA) was performed according to product manual. The distribution of visible black spots representing the potential regulatory factors of LeSPL-CNR on the PVDF membrane was photographed. The total experiment was performed three times independently, and the spots detected at least twice at same position were considered as candidates. Then, the corresponding proteins at same positions on duplicated 2-DE gel were manually excised and sent to Sangon Biotech (Shanghai) Co., Ltd for identification by mass spectrometry. The in-gel protein digestion and analysis of matrix assisted laser desorption ionization-time of flight mass spectrometry (MALDI-TOF-MS) were performed using a service from Sangon Biotech (Shanghai) Co., Ltd. MS data acquired on an AB SCIEX TOF/TOF 5800 system (Applied Biosystems, USA) were used to search against NCBInr protein databases (http://www.ncbi.nlm.nih.gov) with Mascot MS/MS Ions Search program on the Matrix Science public web site

(http://www.matrixscience.com). Search parameters were set as proteolytic enzyme, trypsin; max missed cleavages, 1; fixed modifications, carbamidomethyl (C); variable modifications, oxidation $(\mathrm{M})$; peptide mass tolerance, $1.2 \mathrm{Da}$; and fragment mass tolerance, $0.6 \mathrm{Da}$. Only significant hits as defined by Mascot 
probability analysis were considered.

\subsection{Yeast One-Hybrid (Y1H) Screening}

A Matchmaker Gold Yeast One-Hybrid Library Screening System (Clontech, USA) was used as described by the manufacturer. Briefly, the $286 \mathrm{bp}$ fragment was amplified using a pair of primers (P2F and P2R in Table 1), sequenced, and cloned into the pAbAi vector in digestion sites of restriction enzymes HindIII/SaIl. After linearizing by BstBI, the pBait-AbAi plasmid was transformed to Y1HGold strain following the protocol of the Yeastmaker Yeast Transformation System 2 (Clontech, USA) to generate bait yeast strain. Then, the working concentration of Aureobasidin A ( $\mathrm{AbA}$ ) used to suppress basal expression of our bait construct in the absence of prey was determined on SD/-Ura media with different concentrations of AbA.

Total RNA was extracted from AC fruits at breaker stage using an RNeay Plant Mini Kit (Qiagen, Germany). The first-strand SMART cDNA synthesis, amplifying SMART (Switching Mechanism at 5' end of RNA Transcript) cDNA by long distance PCR, and purifying the ds CDNA with a CHROMA SPIN + TE-400 column (Clontech, USA) were performed following protocol of Clontech's SMART technology. The purified SMART ds cDNA and pGADT7-Rec AD Cloning Vector (SmaI-linearized) were co-transformed into bait yeast strain using the Yeastmaker Yeast Transformation System 2. The aliquot suspensions of the library transformation reaction were dispersed evenly on the $150 \mathrm{~mm}$ plates with $\mathrm{SD} /-\mathrm{Leu} / \mathrm{ABA}^{100}$. After incubation for $3-5$ days, the positive clones were isolated and used to extract prey plasmids responsible for activation of reporters by a TIANprep Yeast Plasmid DNA Kit (Tiangen, China). At last, cDNA inserts from pGADT7-Rec vectors were identified by PCR amplification with T7 sequencing primer (T7 SP) and 3'AD sequencing primer (3'AD SP) (Table 1) according to the protocol of A Matchmaker Gold Yeast One-Hybrid Library Screening System, and sequencing in Shanghai Sunny Biotechnology Co., Ltd. Sequencing results were analyzed on https://blast.ncbi.nlm.nih.gov/Blast.cgi. Search parameters were set as Database: Nucleotide collection (nr/nt); Organism: Solanum lycopersicum; Exclude: Uncultured/environmental sample sequences; Limit to: No; Entrez Query: No.

Table 1. Primers used in this study.

\begin{tabular}{ccc}
\hline Name & Sequence & Annealing temperature $\left({ }^{\circ} \mathrm{C}\right)$ \\
\hline P1F & CAGTAATAAGAAACAAGTTATTTCAAC & 53 \\
P1R & GAACCGGAAGTGTCAGATTAAAACAC & \\
P2F & GCTACCAAGCTTCAGTAATAAGAAACAAGTTATTTC & 55 \\
P2R & GAGTGCGTCGACGAACCGGAAGTGTCAGATTAAAAC & \\
T7 SP & TAATACGACTCACTATAGGGC & 55 \\
3'AD SP & AGATGGTGCACGATGCACAG & \\
\hline
\end{tabular}




\section{Results}

\subsection{Cis-Acting Regulatory Element Analysis in Specific 286 bp Region}

In this study, potential cis-acting regulatory elements of the specific 286 bp region were analyzed online in PlantCARE database. Total eight kinds of motifs (including AE-box, CAAT-box, CCAAT-box, HSE, TATA-box, TCCACCT-motif, WUN-motif and unname-4 motif) were forecasted in both forward and reverse complement sequences (Figure 1(c)). These motifs were composed of 135 nucleotides which accounted for about $47 \%$ of the 286 bp region. Unexpectedly, most of the methylated cytosine residues in the $286 \mathrm{bp}$ region did not showed a correlation with these motifs. These results indicated that the $286 \mathrm{bp}$ region possessed structural basis for potential regulators binding.

\subsection{Screening Potential Regulators by South-Western Blotting}

The DIG system is a simple, effective system for nonradioactive labeling and detection of nucleic acids. In this study, the polymerase chain reaction was performed for incorporation of DIG-11-dUTP into the specific 286 bp probe (Figure 2(a)). The labeling efficiency was determined by comparison to the DIG-labeled control DNA. And $1 \mathrm{ng} / \mu \mathrm{L}$ of DIG-labeled DNA probe was used as

(a)

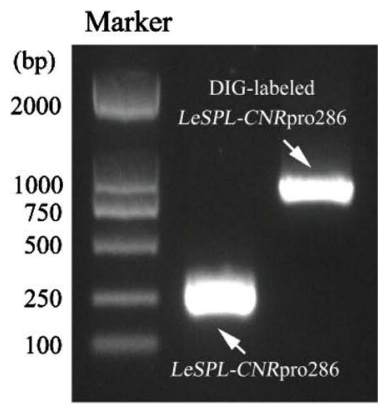

(b)

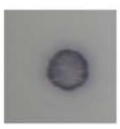

10
DIG-labeled pBR328 DNA (pg/ $\mu \mathrm{L})$

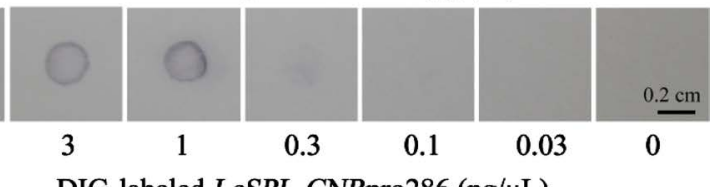

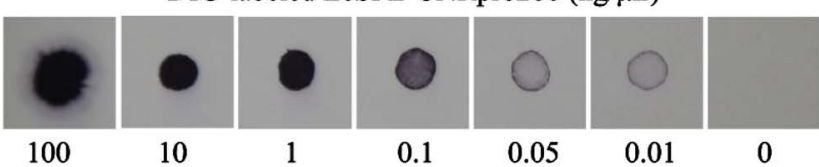

Concentration

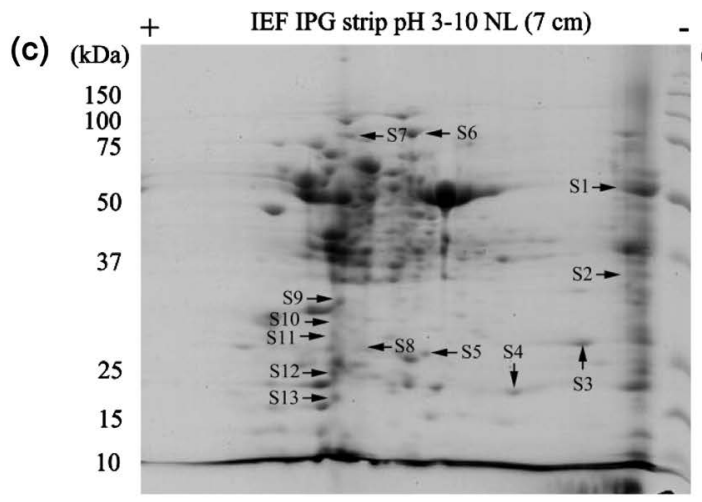

2-DE gel

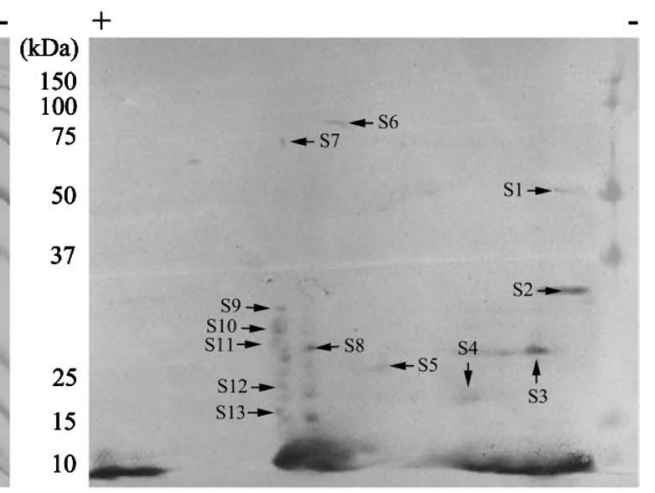

PVDF membrane

Figure 2. Digoxigenin-labeled LeSPL-CNRpro286 probes (a), determination of labeling efficiency (b), and $L e S P L-C N R$ regulatory factor screening by two-dimensional electrophoresis (2-DE) combined with south-western blotting analysis (c). Spots indicated by black arrow in 2-DE gel and PVDF membrane were potential regulatory factors which could bind with DIG-labeled LeSPL-CNRpro286 probes. The spots were numbered and identified by MS/MS, corresponding to those in Table 2. 
working concentration in the subsequent hybridization detection (Figure 2(b)). Proteins extracted from tomato fruits at breaker stage were separated using 2-DE. More than 300 protein spots were detected in the gel after ignoring very faint spots and spots with undefined shapes by Image Master 2D Elite software. After south-western blotting detection, a total of 20 spots on the PVDF membrane were considered as positive signals (Figure $2(\mathrm{c})$ ), and the corresponding proteins were identified by mass spectrometry and were matched in NCBInr database. Among them, 13 proteins were matched to proteins of Solanum lycopersicum with known or predicted functions (Table 2) including elongation factor 1-alpha, basic $30 \mathrm{kDa}$ endochitinase precursor, osmotin-like protein precursor, ribulose-1,5-bisphosphate carboxylase/oxygenase large subunit, etc.

\subsection{Screening Potential Regulators by Yeast One-Hybrid Library Screening System}

Yeast one-hybrid system is a powerful technique to rapidly identify heterologous proteins that can interact with a specific regulatory DNA sequence of interest (the bait sequence) and uses a single fusion protein in which the activation domain (AD) is linked directly to the binding domain. The binding domain in this case also may be constituted by a library. In this study, our target-reporter

Table 2. Proteins information acquired through south-western blotting combined with MALDI-TOF-MS analysis.

\begin{tabular}{|c|c|c|c|c|c|c|c|}
\hline Number & Name & NCBI accession ${ }^{\mathrm{a}}$ & $\begin{array}{l}\text { Theo. } \\
\text { Mass/PI }\end{array}$ & Species & $\begin{array}{c}\text { Mascot } \\
\text { Score/Threshold }\end{array}$ & $\begin{array}{l}\mathrm{SC} \\
(\%)^{\mathrm{d}}\end{array}$ & $\mathrm{NP}^{\mathrm{e}}$ \\
\hline S1 & Elongation factor 1-alpha & NP_001234035 & $49613 / 9.24$ & Solanum lycopersicum & $345 / 45$ & 12 & $4 / 4$ \\
\hline S2 & Basic $30 \mathrm{kDa}$ endochitinase precursor & NP_001234403 & $33209 / 8058$ & Solanum lycopersicum & $356 / 43$ & 16 & $3 / 3$ \\
\hline S3 & Osmotin-like protein precursor & NP_001234714.1 & $28159 / 8.15$ & Solanum lycopersicum & $151 / 44$ & 10 & $2 / 2$ \\
\hline S4 & $\begin{array}{l}\text { Ribulose-1,5-bisphosphate } \\
\text { carboxylase/oxygenase large subunit }\end{array}$ & AAA19771.1 & $53398 / 6.55$ & Solanum lycopersicum & $69 / 45$ & 2 & $1 / 1$ \\
\hline S5 & Predicted triosephosphate isomerase & XP_004236746.1 & $27251 / 5.73$ & Solanum lycopersicum & $390 / 44$ & 20 & $4 / 4$ \\
\hline S6 & $\begin{array}{l}\text { Predicted 5-methyltetrahydropteroyltriglutamate } \\
\text { homocysteine methyltransferase }\end{array}$ & XP_004249374 & $84898 / 5.93$ & Solanum lycopersicum & $1200 / 44$ & 16 & 9/9 \\
\hline S7 & Predicted transketolase & XP_004248560.1 & $80341 / 5.94$ & Solanum lycopersicum & $677 / 43$ & 12 & $6 / 6$ \\
\hline S8 & Cytosolic ascorbate peroxidase 1 & NP_001234782.1 & $27733 / 5.61$ & Solanum lycopersicum & $622 / 44$ & 34 & $6 / 6$ \\
\hline S9 & Oxygen-evolving enhancer protein 1 & NP_001296294.1 & $25377 / 5.89$ & Solanum lycopersicum & $250 / 44$ & 11 & $2 / 2$ \\
\hline S10 & $40 \mathrm{~S}$ ribosomal protein $\mathrm{S} 8$ & XP_004242427.1 & $24847 / 10.39$ & Solanum lycopersicum & $196 / 44$ & 12 & $2 / 2$ \\
\hline S11 & Predicted triosephosphate isomerase & XP_012084383.1 & $33880 / 6.13$ & Solanum lycopersicum & $225 / 45$ & 9 & $2 / 2$ \\
\hline S12 & $\begin{array}{l}\text { Predicted ribulose-phosphate 3-epimerase, } \\
\text { cytoplasmic isoform-like }\end{array}$ & XP_004238785.1 & $23944 / 5.29$ & Solanum lycopersicum & $99 / 43$ & 6 & $1 / 1$ \\
\hline S13 & Predicted: adenine phosphoribosyltransferase 4 & XP_004238096.2 & $19993 / 5.17$ & Solanum lycopersicum & $389 / 45$ & 20 & $4 / 4$ \\
\hline
\end{tabular}

${ }^{a} \mathrm{NCBI}$ Accession: the accession number of non-redundant protein database; ${ }^{\mathrm{b}}$ Theo. Mass/PI: theoretical molecular mass and isoelectric point based on amino acid sequence of the identified protein; ${ }^{\mathrm{c}}$ Mascot Score/Threshold: Mascot scores are statistically significant $(\mathrm{P}<0.05)$; ${ }^{\mathrm{d} S C}$ : Amino acid sequence coverage for the identified proteins; ${ }^{\mathrm{e}} \mathrm{NP}$ : The total number of matched peptides/the number of matched peptides whose mascot scores are statistically significant $(\mathrm{P}<0.05)$. 
construct (pBait-AbAi) contained one copy of the specific 286 bp sequence inserted in the upstream of $A b A^{r}$ reporter gene in pAbAi (Figure 3(a)). After detection of basal expression of the bait reporter in the absence of prey, $100 \mathrm{ng} / \mathrm{mL}$ was consider as minimal inhibitory concentration of $\mathrm{AbA}$ and used in the subsequent library screening. The cDNA library of AC fruit at breaker stage were constructed by SMART technology (Figure 3(b) and Figure 3(c)) and the number of screened clones was about 0.3 million by counting the number of colonies on SD/-Leu. Meanwhile, far fewer (about 80) colonies, which contained targeted prey plasmid as well as the positive interaction of the potential regulatory factors with the specific 286 bp region, were acquired on SD/-Leu/AbA ${ }^{100}$. After confirming reporter phenotype by restreaking onto fresh selective media (Figure 3(d)), yeast colony PCR, rescue and isolation of the library plasmid responsible for activation of reporters, a total of 28 genuine positive colonies were
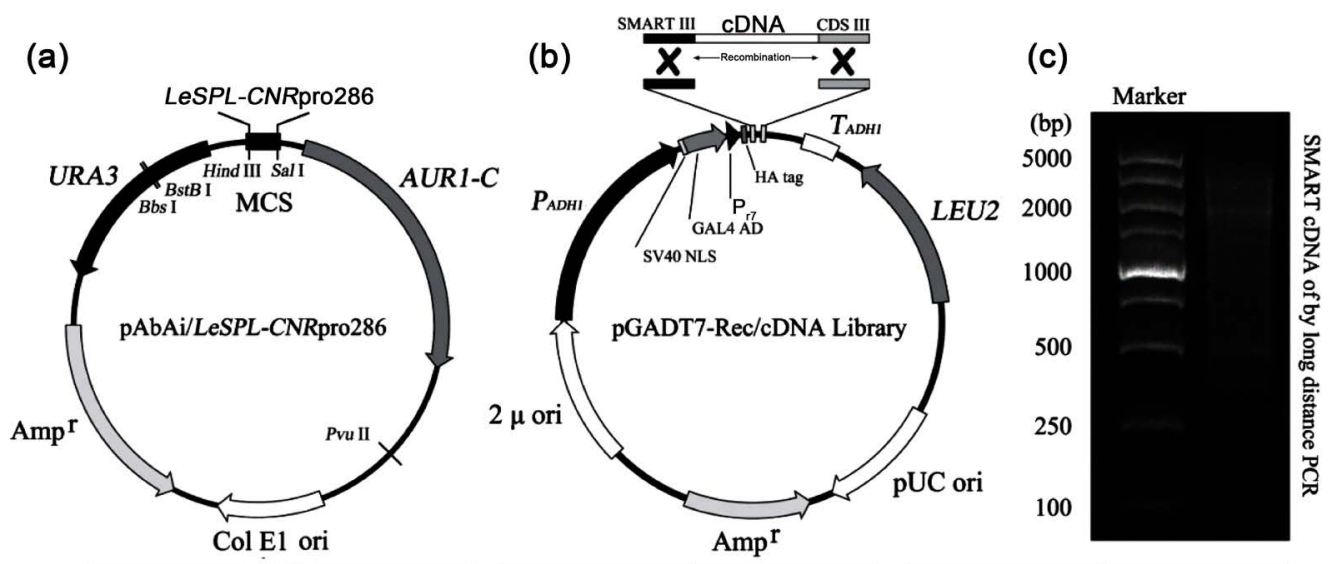

(d)
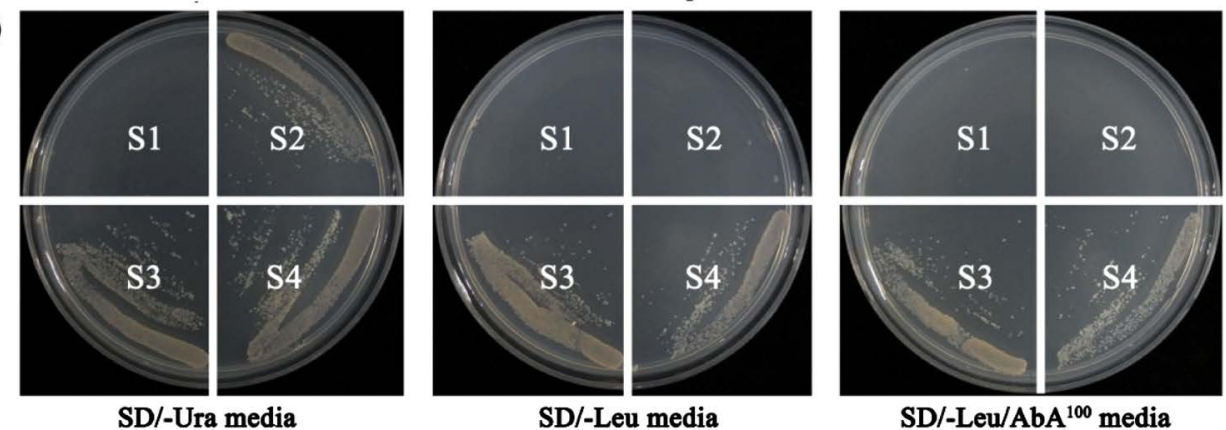

Figure 3. LeSPL-CNR regulatory factors screening by yeast one-hybrid library screening system. A 286 bp region of the $L E S P L-C N R$ promoter was cloned into the pAbAi reporter vector which was then integrated into the Y1HGold genome to create a bait-specific reporter strain (a). Activation of the $\mathrm{AbA}$ resistance gene $\left(\mathrm{AbA}^{\mathrm{r}}\right)$ occurred if a potential regulatory factor could bind to the bait sequence. A cDNA library from tomato fruit at breaker stage was created by SMART cDNA synthesis technology (c) and the cDNA pool with flanking end sequences were homologous to the prey vector, pGADT7-Rec (b). When the cDNA and linear prey vector were transformed into the reporter strain together, the yeast recombined the cDNA and the vector. Transformed cells were plated on SD/-Ura, $\mathrm{SD} /$-Leu and $\mathrm{SD} /$-Leu/AbA ${ }^{100}$ media to determine if pAbAi report vector or pGADT7-Rec existed in the cells, and select positive colonies which had activated the $\mathrm{AbA}^{\mathrm{r}}$ reporter (d). S1: Y1HGold wild type strain, as a negative control; S2: Y1HGold [pAbAi/LeSPL-CNRpro286] strain; S3: Y1HGold [pGADT7-Rec/p53, pAbAi/p53] as a positive control; S4: Y1HGold [pGADT7-Rec/BP1, pAbAi/LeSPL-CNRpro286]. 
distinguished from false positive colonies. Finally, a total of 19 prey inserts were identified by sequencing and the detailed information of these candidates was shown in Table 3.

\section{Discussion}

Gene expression regulatory networks are comprised of cis- and trans-acting factors, and differences in gene expression are attributable to genetic variation. Epigenetic change can also be considered as an important factor in understanding phenotypic change. LeSPL-CNR of Solanum lycopersicum is an excellent research subject which possesses important biological functions and is controlled by both epigenetic and classical genetics approaches. In this study, a 286 bp contiguous region at $2.4 \mathrm{~kb}$ upstream from the LeSPL-CNR coding sequence was used as a bait to search potential regulatory factors by south-western blotting and yeast one-hybrid library screening system. A total of 32 candidate proteins were acquired, and they distributed in nucleus, membrane, mitochondrial, cytoplasm and chloroplast. Except three uncharacterized candidates (BP12, BP15 and BP19), other candidates have known or predicted functions in an extensive range including structural protein, enzyme, and cell signaling, etc. Among them,

Table 3. Information of sequences received from yeast one-hybrid library screening system.

\begin{tabular}{|c|c|c|c|}
\hline Number & NCBI accession & Species & Description \\
\hline BP1 & NM_001247170 & Solanum lycopersicum & Alcohol dehydrogenase \\
\hline BP2 & XM_004247745.2 & Solanum lycopersicum & Predicted: putative G3BP-like protein-like \\
\hline BP3 & XM_004246323.2 & Solanum lycopersicum & Predicted: phosphatidylinositol: ceramide inositolphosphotransferase 2 \\
\hline BP4 & XM_004247148.2 & Solanum lycopersicum & Predicted: GDP-mannose transporter GONST4 \\
\hline BP5 & XM_004242375.2 & Solanum lycopersicum & Predicted: peptidyl-prolyl cis-trans isomerase FKBP62-like \\
\hline BP6 & XM_004241364.2 & Solanum lycopersicum & Predicted: chloroplast stem-loop binding protein of $41 \mathrm{kDa} \mathrm{b}$ \\
\hline BP7 & NM_001308004.1 & Solanum lycopersicum & Predicted: mitochondrial CBS domain-containing protein CBSX3 \\
\hline BP8 & XM_004249296.2 & Solanum lycopersicum & Predicted: calmodulin-7 \\
\hline BP9 & XM_004229193.2 & Solanum lycopersicum & Predicted: 40S ribosomal protein S21-2-like \\
\hline BP10 & XM_004229308.2 & Solanum lycopersicum & Predicted: histone $\mathrm{H} 3.2$ \\
\hline BP11 & XM_004245431.2 & Solanum lycopersicum & Predicted: $\mathrm{ABC}$ transporter $\mathrm{F}$ family member 3 \\
\hline BP12 & XM_004250280.2 & Solanum lycopersicum & Uncharacterized LOC101249421 \\
\hline BP13 & XM_004250067.2 & Solanum lycopersicum & Predicted: histone H3.3 \\
\hline BP14 & NM_001308944.1 & Solanum lycopersicum & Chloroplastic ribulose bisphosphate carboxylase small chain $2 \mathrm{~A}$ \\
\hline BP15 & AK324254.1 & Solanum lycopersicum & LEFL1074DA11, HTC in leaf \\
\hline BP16 & XM_004230295 & Solanum lycopersicum & Predicted: shaggy-related protein kinase NtK-1 \\
\hline BP17 & XM_004232376.2 & Solanum lycopersicum & Predicted: mitochondrial-like Gamma carbonic anhydrase 1, \\
\hline BP18 & XM_004251215.2 & Solanum lycopersicum & Predicted: ATP synthase subunit $\mathrm{d}$, mitochondrial-like \\
\hline BP19 & AK319554.1 & Solanum lycopersicum & LEFL1043BF01, HTC in leaf \\
\hline
\end{tabular}


BP10 and BP13 were predicted as histone H3.2 and H3.3, S4 and BP14 belonged to Ribulose-1,5-bisphosphate carboxylase/oxygenase, and S10 and BP9 belonged to $40 \mathrm{~S}$ ribosomal protein. Same results acquired from same or different approaches indicated that screening methods in this study were feasible and available.

It is known that histone $\mathrm{H} 3$ is one of five main histone proteins involved in the structure of chromatin in eukaryotic cell. Featuring a main globular domain and a long N-terminal tail, $\mathrm{H} 3$ is involved with the structure of the nucleosomes on the beads on a string structure. Its sequence variants and variable modification stages are thought to play a role in the dynamic and long term regulation of genes [34]. Variant H3.2 (BP10) closely with H3.1 (only differing in a Cys-Ser substitution at amino acid position) is coupled to DNA synthesis during DNA replication and possibly DNA repair, whereas histone variant $\mathrm{H} 3.3$ (BP13) is necessary to mediated DNA-synthesis-dependent and -independent nucleosome assembly [35]. Therefore, the interaction between $286 \mathrm{bp}$ fragment and Histone $\mathrm{H} 3$ is reasonable.

Calmodulin (BP8), an abbreviation for calcium-modulated protein, is a multifunctional intermediate calcium-binding messenger protein expressed in eukaryotic cells. It is a small highly conserved protein that is 148 amino acids long (16.7 $\mathrm{kDa}$ ). This protein has two approximately symmetrical globular domains each containing a pair of EF-hand motif separated by a flexible linker region for a total of four $\mathrm{Ca}^{2+}$ binding sites. Once bound to $\mathrm{Ca}^{2+}$, calmodulin acts as part of a signal transduction pathway by modifying its interactions with various target proteins such as kinases or phosphatases [36]. Calmodulin can undergo post-translation modifications, such as phosphorylation, acetylation, methylation and proteolytic cleavage, each of which has potential to modulate its actions [37]. For absence of clues about connection between calmodulin and the 286 bp fragment, a possibility is that calmodulin might form a complex with other protein or proteins to fulfil the regulatory responsibilities. This mode can refer the report of Shima et al. [38] that tomato RIN forms a complex with FUL1 and FUL2, and this complex can bind to a typical target DNA motif for MADS-box proteins.

Petidyl-prolyl cis-trans isomerase (BP5), as another candidate, is an enzyme that interconverts the cis and trans isomers of peptide bonds with the amino acid proline (http://www.uniprot.org/uniprot/P62937). Proline has an unusually conformationally restrained peptide bond duo to its cyclic structure with its side chain bonded to its secondary amine nitrogen. This structure can overcome the energetic preference for the trans peptide bond conformation and stabilize the cis form so that both isomers are populated under biologically relevant conditions. The process of cis-trans isomerization can be the rate-limiting step in the process of protein folding, and petidyl-prolyl cis-trans isomerase therefore functions as protein folding chaperones [39]. It probably makes petidyl-prolyl cis-trans isomerase participate in transcription regulation of LeSPL-CNR in an indirect way.

Up to now, there is insufficient evidence confirming the positive correlations 
between other candidate proteins not mentioned and transcription regulation of LeSPL-CNR. For example, 5-methyltetrahydropteroyltriglutamate homocysteine methyltransferase (S6) belongs to the family of transferases which catalyzes the transfer of a methyl group from 5-methyltetrahydrofolate to homocysteine resulting in methionine formation (http://enzyme.expasy.org/EC/2.1.1.14); Transketolase (S7) is a ubiquitous enzyme that catalyzes the reversible transfer of a two-carbon ketol group from fructose-6-phosphate or sedoheptulose-7-phosphate to glyceraldehyde-3-phosphate to yield xylulose-5-phosphate and erythrose-4-phosphate or ribose-5-phosphate [40]. These two proteins involve in methionine metabolism and Calvin cycle, respectively. Therefore, further exploration about function of the 286 bp region via multiple approaches from different aspects is necessary. Furthermore, it has a potential of obtaining more valuable information and excluding the false positive results via optimizing experiment conditions.

\section{Conclusion}

In this study, potential regulators of LeSPL-CNR, which could bind to the specific 286 bp region of LeSPL-CNR promoter, were screened via south-western blotting and yeast one-hybrid library screening system. A total of 13 and 19 candidate proteins were acquired respectively. Although current knowledge could not reasonably explain the molecular mechanism of interactions between acquired candidates and $286 \mathrm{bp}$ fragment, results still provided some interesting information for further exploration in transcriptional cascade or epigenetic controls for fruit ripening.

\section{Acknowledgements}

This work was supported by the Zhejiang Provincial Natural Science Foundation of China (LY18C150009) and the National Natural Science Foundation of China (31501810, 31401926).

\section{References}

[1] Giovannoni, J.J. (2004) Genetic Regulation of Fruit Development and Ripening. Plant Cell, 16, 170-180. https://doi.org/10.1105/tpc.019158

[2] Llorente, B., D’Andrea, L., Rui-Sola, M.A., Botterweg, E., Pulido, P., Andilla, J., Loza-Alvarez, P. and Rodriguez-Concepcion, M. (2016) Tomato Fruit Carotenoid Biosynthesis Is Adjusted to Actual Ripening Progression by a Light-Dependent Mechanism. Plant Journal, 85, 107-119. https://doi.org/10.1111/tpj.13094

[3] Qin, G.Z., Zhu, Z., Wang, W.H., Cai, J.H., Chen, Y., Li, L. and Tian, S.P. (2016) A Tomato Vacuolar Invertase Inhibitor Mediates Sucrose Metabolism and Influences Fruit Ripening. Plant Physiology, 172, 1596-1611. https://doi.org/10.1104/pp.16.01269

[4] The Tomato Genome Consortium (2012) The Tomato Genome Sequence Provides Insights into Fleshy Fruit Evolution. Nature, 485, 635-641. https://doi.org/10.1038/nature11119

[5] Kudo, T., Kobayashi, M., Terashima, S., Katayama, M., Ozaki, S., Kanno, M., Saito, M., Yokoyama, K., Ohyanagi, H., Aoki, K., Kubo, Y. and Yano, K. (2017) Toma- 
tomics: A Web Database for Integrated Omics Information in Tomato. Plant Cell and Physiology, 58, e8. https://doi.org/10.1093/pcp/pcw207

[6] Cara, B. and Giovannoni, J.J. (2008) Molecular Biology of Ethylene during Tomato Fruit Development and Maturation. Plant Science, 175, 106-113. https://doi.org/10.1016/j.plantsci.2008.03.021

[7] Bapat, V.A., Trivedi, P.K., Ghosh, A., Sane, V.A., Ganapathi, T.R. and Nath, P. (2010) Ripening of Fleshy Fruit: Molecular Insight and the Role of Ethylene. Biotechnology Advances, 28, 94-107. https://doi.org/10.1016/j.biotechadv.2009.10.002

[8] Gapper, N.E., McQuinn, R.P. and Giovannoni, J.J. (2013) Molecular and Genetic Regulation of Fruit Ripening. Plant Molecular Biology, 82, 575-591.

https://doi.org/10.1007/s11103-013-0050-3

[9] Mou, W.S., Li, D.D., Bu, J.W., Jiang, Y.Y., Khan, Z.U., Luo, Z.S., Mao, L.C. and Ying, T.J. (2016) Comprehensive Analysis of ABA Effects on Ethylene Biosynthesis and Signaling during Tomato Fruit Ripening. PLoS ONE, 11, e0154072.

https://doi.org/10.1371/journal.pone.0154072

[10] Wang, R.H., Yuan, X.Y., Meng, L.H., Zhu, B.Z., Zhu, H.L., Luo, Y.B. and Fu, D.Q. (2016) Transcriptome Analysis Provides a Preliminary Regulation Route of the Ethylene Signal Transduction Component, SIEIN2, during Tomato Ripening. PLoS ONE, 11, e0168287. https://doi.org/10.1371/journal.pone.0168287

[11] Rohrmann, J., McQuinn, R., Giovannoni, J.J., Fernie, A.R. and Tohge, T. (2012) Tissue Specificity and Differential Expression of Transcription Factors in Tomato Provide Hints of Unique Regulatory Networks during Fruit Ripening. Plant Signalign \& Behavior, 7, 1639-1647. https://doi.org/10.4161/psb.22264

[12] Arhondakis, S., Bita, C.E., Perrakis, A., Manioudaki, M.E., Krokida, A., Kaloudas, D. and Kalaitzis, P. (2016) In silico Transcriptional Regulatory Networks Involved in Tomato Fruit Ripening. Frontiers in Plant Science, 7, 1234. https://doi.org/10.3389/fpls.2016.01234

[13] Manning, K., Tör, M., Poole, M., Hong, Y.G., Thompson, A.J., King, G.J., Giovannoni, J.J. and Seymour, G.B. (2006) A Naturally Occurring Epigenetic Mutation in a Gene Encoding an SBP-Box Transcription Factor Inhibits Tomato Fruit Ripening. Nature Genetics, 38, 948-952. https://doi.org/10.1038/ng1841

[14] Lin, Z.F., Hong, Y.G. and Yin, M.G. (2008) A Tomato HD-Zip Homeobox Protein, LeHB-1, Plays an Important Role in Floral Organogenesis and Ripening. Plant Journal, 55, 301-310. https://doi.org/10.1111/j.1365-313X.2008.03505.X

[15] Vrebalov, J., Pan, I.L., Arroyo, A.J.M., McQuinn, R., Chung, M., Poole, M., Rose, J., Seymour, G., Grandillo, S., Giovannoni, J. and Irish, V.F. (2009) Fleshy Fruit Expansion and Ripening Are Regulated by the Tomato SHATTERPROOF Gene TAGL1. Plant Cell, 21, 3041-3062. https://doi.org/10.1105/tpc.109.066936

[16] Karlova, R., Rosin, F.M., Busscher-Lange, J., Parapunova, V., Do, P.T., Fernie, A.R., Fraser, P.D., Baxter, C., Angenent, G.C. and De Maagd, R.A. (2011) Transcriptome and Metabolite Profiling Show That APETALA2a Is a Major Regulator of Tomato Fruit Ripening. Plant Cell, 23, 923-941. https://doi.org/10.1105/tpc.110.081273

[17] Dong, T.T., Hu, Z., Deng, L., Wang, Y., Zhu, M.K., Zhang, J.L. and Chen, G.P. (2013) A Tomato MADS-Box Transcription Factor, SIMADS1, Acts as a Negative Regulator of Fruit Ripening. Plant Physiology, 163, 1026-1036. https://doi.org/10.1104/pp.113.224436

[18] Fujisawa, M., Nakano, T., Shima, Y. and Yasuhiro, I. (2013) A Larger-Scale Identification of Direct Targets of the Tomato MADS Box Transcription Factor Ripening Inhibitor Reveals the Regulation of Fruit Ripening. Plant Cell, 25, 371-386. 
https://doi.org/10.1105/tpc.112.108118

[19] Fujisawa, M., Shima, Y., Nakagawa, H., Kitagawa, M., Kimbara, J., Nakano, T., Kasumi, T. and Ito, Y. (2014) Transcriptional Regulation of Fruit Ripening by Tomato Fruitfull homologs and Associated MADS Box Proteins. Plant Cell, 26, 89-101. https://doi.org/10.1105/tpc.113.119453

[20] Zhu, M.K., Chen, G.P., Zhou, S., Tu, Y., Wang, Y., Dong, T.T. and Hu, Z.L. (2014) A New Tomato NAC (NAM/ATAF1/2/CUC2) Transcription Factor, SINAC4, Fuctions as a Positive Regulator of Fruit Ripening and Carotenoid Accumulation. Plant Cell and Physiology, 55, 119-135. https://doi.org/10.1093/pcp/pct162

[21] Weng, L., Zhao, F.F., Li, R., Xu, C.J., Chen, K.S. and Xiao, H. (2015) The Zinc Finger Transcription Factor SIZFP2 Negatively Regulates Abscisic Acid Biosynthesis and Fruit Ripening in Tomato. Plant Physiology, 167, 931-949.

https://doi.org/10.1104/pp.114.255174

[22] Niederhuth, C.E. and Schmitz, R.J. (2014) Covering Your Bases: Inheritance of DNA Methylation in Plant Genomes. Molecular Plant, 7, 472-480.

https://doi.org/10.1093/mp/sst165

[23] Kasai, A. and Harada, T. (2015) Epimutant Induction as a New Plant Breeding Technology. JARQ—Japan Agricultural Research Quarterly, 49, 301-305.

https://doi.org/10.6090/jarq.49.301

[24] Liu, R.E., How-Kit, A., Stammitti, L., Teyssier, E., Rolin, D., Mortain-Bertrand, A., Halle, S., Liu, M.C., Kong, J.H., Wu, C.Q., Degraeve-Guibault, C., Chapman, N.H., Maucourt, M., Hodgman, T.C., Tost, J., Bouzayen, M., Hong, Y.G., Seymour, G.B., Giovannoni, J.J. and Gallusci, P. (2015) A DEMETER-Like DNA Demethylase Governs Tomato Fruit Ripening. Proceeding of the National Academy of Science of the United States of America, 112, 10804-10809.

https://doi.org/10.1073/pnas.1503362112

[25] Zhong, S., Fei, Z.J., Chen, Y.R., Zheng, Y., Huang, M.Y., Vrebalov, J., McQuinn, R., Gapper, N., Liu, B., Xiang, J., Shao, Y. and Giovannoni, J.J. (2013) Single-Base Resolution Methylomes of Tomato Fruit Development Reveal Epigenome Modifications Associated with Ripening. Nature Biotechnology, 31, 154-161. https://doi.org/10.1038/nbt.2462

[26] Chen, W.W., Kong, J.H., Qin, C., Yu, S., Tan, J.J., Chen, Y.R., Wu, C.Q., Wang, H., Shi, Y., Li, C.Y., Zhang, P.C, Wang, Y., Lai, T.F., Yu, Z.M., Zhang, X., Shi, N.N., Wang, H.Z., Osman, T., Liu, Y.L., Manning, K., Jackson, S., Rolin, D., Zhong, S.L., Seymour, G.B., Gallusci, P. and Hong, Y. (2015) Requirement of Chromomethylase3 for Somatic Inheritance of the Spontaneous Tomato Epimutation Colourless Non-Ripening. Scientific Reports, 5, Article No. 9192.

https://doi.org/10.1038/srep09192

[27] Long, M., Millar, D.J., Kimura, Y., Donovan, G., Rees, J., Fraser, P.D., Bramley, P.M. and Bolwell, G.P. (2006) Metabolite Profiling of Carotenoid and Phenolic Pathway in Mutant and Transgenic Lines of Tomato: Identification of a High Antioxidant Fruit Line. Phytochemistry, 67, 1750-1757.

https://doi.org/10.1016/j.phytochem.2006.02.022

[28] Orfila, C., Huisman, M.M.H., Willats, W.G.T., van Alebeek, G.W.M., Schols, H.A., Seymour, G.B. and Knox, J.P. (2002) Altered Cell Wall Disassembly during Ripeing of Cnr Tomato Fruit: Implications for Cell Adhesion and Fruit Softening. Planta, 215, 440-447. https://doi.org/10.1007/s00425-002-0753-1

[29] Chen, W.W., Kong, J.H., Lai, T.F., Manning, K., Wu, C.Q., Wang, Y., Qin, C., Li, B., Yu, Z., Zhang, X., He, M.L., Zhang, P.C., Gu, M., Yang, X., Mahammed, A., Li, C.Y., 
Osman, T., Shi, N.N., Wang, H.Z., Jackson, S., Liu, Y., Gollusci, P. and Hong, Y. (2015) Tuning LeSPL-CNR Expression by SlymiR157 Affects Tomato Fruit Ripening. Scientific Reports, 5, Article No. 7852. https://doi.org/10.1038/srep07852

[30] Kanazawa, A., Inaba. J., Shimura, H., Otagaki, S., Tsukahara, S., Matsuzawa, A., Kim, B.M., Goto, K. and Masuta, C. (2011) Virus-Mediated Efficient of Epigenetic Modifications of Endogenous Genes with Phenotypic Changes in Plants. Plant Journal, 65, 156-168. https://doi.org/10.1111/j.1365-313X.2010.04401.X

[31] Lescot, M., Déhais, P., Thijs, G., Marchal, K., Moreau, Y., De Peer, Y.V., Rouzé, P. and Rombauts, S. (2002) PlantCARE, a Database of Plant Cis-Acting Regulatory Elements and a Portal to Tools for in Silico Analysis of Promoter Sequences. Nucleic Acids Research, 30, 325-327. https://doi.org/10.1093/nar/30.1.325

[32] Qin, G.Z., Wang, Y.Y., Cao, B.H., Wang, W.H. and Tian, S.P. (2012) Unraveling the Regulatory Network of the MADS Box Transcription Factor RIN in Fruit Ripening. Plant Journal, 70, 243-255. https://doi.org/10.1111/j.1365-313X.2011.04861.x

[33] Qin, G.Z., Tian, S.P., Chan, Z.L. and Li, B.Q. (2007) Crucial Role of Antioxidant Proteins and Hydrolytic Enzymes in Pathogenicity of Penicillium expansum: Analysis Based on Proteomic Approach. Molecular \& Cellular Proteomics, 6, 425-438. https://doi.org/10.1074/mcp.M600179-MCP200

[34] Jacob, Y., Bergamin, E., Donoghue, M.T.A., Mongeon, V., LeBlanc, C., Voigt, P., Underwood, C.J., Brunzelle, J.S., Michaels, S.D., Reinberg, D., Couture, J.F. and Martienssen, R.A. (2014) Selective Methylation of Histone H3 Variant H3.1 Regulates Heterochromatin Replication. Science, 343, 1249-1253. https://doi.org/10.1126/science.1248357

[35] Tagami, H., Ray-Gallet, D., Almouzni, G. and Nakatani, Y. (2004) Histone H3.1 and H3.3 Complexes Mediate Nucleosome Assembly Pathways Dependent or Independent of DNA Synthesis. Cell, 116, 51-61. https://doi.org/10.1016/S0092-8674(03)01064-X

[36] Chin, D. and Means, A.R. (2000) Calmodulin: A Prototypical Calcium Sensor. Trends in Cell Biology, 10, 322-328. https://doi.org/10.1016/S0962-8924(00)01800-6

[37] Banerjee, J., Magnani, R., Nair, M., Dirk, L.M., BeBolt, S., Maiti, I.B. and Houtz, R.L. (2013) Calmodulin-Mediated Signal Transduction Pathways in Arabidopsis Are Fine-Tuned by Methylation. Plant Cell, 25, 4493-4511. https://doi.org/10.1105/tpc.113.119115

[38] Shima, Y., Kitagawa, M., Fujisawa, M., Nakano, T., Kato, H., Kimbara, J., Kasumi, T. and Ito, Y. (2013) Tomato Fruitefull Homologues Act in Fruit Ripening via Forming MADS-Box Transcription Factor Complexes with RIN. Plant Molecular Biology, 82, 427-438. https://doi.org/10.1007/s11103-013-0071-y

[39] Fischer, G. and Schmid, F.X. (1990) The Mechanism of Protein Folding: Implications of in Vitro Refolding Models for de Novo Protein Folding and Translocation in the Cell. Biochemistry, 29, 2205-2212. https://doi.org/10.1021/bi00461a001

[40] Saha, A., Connelly, S., Jiang, J.J., Zhuang, S.H., Amador, D.T., Phan, T., Pilz, R.B. and Boss, G.R. (2014) Akt Phosphorylation and Regulation of Transketolase Is a Nodal Point for Amino Acid Control of Purine Synthesis. Molecular Cell, 55, 264-276. https://doi.org/10.1016/j.molcel.2014.05.028 